Methods: We used a large Japanese administrative claims database constructed by the Japan Medical Data Center (JMDC) ${ }^{2}$. Patients with the International Classification of Diseases 10th revision (ICD-10) codes for RA were enrolled at the first DMARDs prescription after no DMARDs prescription period for 6-months (index date) in the period from 1/1/2012 to 12/31/2017. Patients who were observable for 12 months after the index date as a follow-up period were included. Patients treated with CSs within the follow-up period were compared with those without them (CS and non-CS group). The primary endpoint was mean medical cost per patient in the 12-month follow-up period. The secondary endpoints were costs for drugs, treatments, and materials and the proportions of patients using the subcategories of each resource. Drugs were divided into medications for RA or for comorbidities including adverse events (AEs). Costs in JPY were converted into EUR (1 EUR = 125 JPY in 2020).

Results: Eligible patients of 1,670 and 1,487 were identified as the CS and non-CS group (median age: 51 years and 50 years). Total mean costs were significantly higher in the CS group (CS, 4,448 EUR, non-CS 3,208 EUR; $P<0.05$ ). Drug, treatment, and material costs were significantly higher in the CS group than in the non-CS group (drug for RA and AEs, CS 2,367 EUR, non-CS 1,581 EUR, $\mathrm{P}<0.05$; drug for RA only, CS 2,265 EUR, non-CS 1,516 EUR, $\mathrm{P}<0.05$; treatment, CS 1,987 EUR, non-CS 1,562 EUR, P < 0.05; material, CS 94 EUR, non-CS 65 EUR; $P<0.05)$. The resource use in almost all drug subcategories were higher in the CS group (Table 1), as well as in all treatment and material subcategories.

Table 1. Number and proportion of patients who used drugs

\begin{tabular}{|c|c|c|c|c|}
\hline \multicolumn{2}{|c|}{ Type of drug } & \multicolumn{3}{|c|}{ Drug use, $n(\%)$} \\
\hline & & $\operatorname{CS}(\mathrm{N}=1,670)$ & Non-CS $(\mathrm{N}=1,487)$ & $P$-value \\
\hline \multirow[t]{3}{*}{ csDMARDs } & Total & $1,635(97.9)$ & 1,447 (97.3) & 0.328 \\
\hline & Methotrexate & $1,481(88.7)$ & $1,315(88.4)$ & 0.870 \\
\hline & Others & 790 (47.3) & $551(37.1)$ & $<0.001$ \\
\hline \multirow[t]{4}{*}{ bDMARDs } & Total & $342(20.5)$ & $181(12.2)$ & $<0.001$ \\
\hline & TNFi & $252(15.1)$ & $129(8.7)$ & $<0.001$ \\
\hline & IL6i & $93(5.6)$ & $40(2.7)$ & $<0.001$ \\
\hline & T-cell & $40(2.4)$ & $17(1.1)$ & 0.012 \\
\hline \multirow[t]{6}{*}{ Analgesics } & Total & $1,512(90.5)$ & $1,274(85.7)$ & $<0.001$ \\
\hline & Acetaminophen & 379 (22.7) & $273(18.4)$ & 0.003 \\
\hline & Acetaminophen / Opioids & $84(5.0)$ & $37(2.5)$ & $<0.001$ \\
\hline & NSAIDs & $1,459(87.4)$ & $1,214(81.6)$ & $<0.001$ \\
\hline & Opioids & $16(1.0)$ & $10(0.7)$ & 0.491 \\
\hline & Others & $198(11.9)$ & $101(6.8)$ & $<0.001$ \\
\hline \multirow[t]{5}{*}{ Antibiotics } & Total & $1,086(65.0)$ & $873(58.7)$ & $<0.001$ \\
\hline & Antibacterial drugs & $1,022(61.2)$ & $800(53.8)$ & $<0.001$ \\
\hline & Antifungal drugs & $133(8.0)$ & $86(5.8)$ & 0.019 \\
\hline & Antiviral drugs & $172(10.3)$ & $129(8.7)$ & 0.136 \\
\hline & Antiparasitic drugs & $5(0.3)$ & $8(0.5)$ & 0.443 \\
\hline \multicolumn{2}{|c|}{ Anti-osteoporotic drugs } & $341(20.4)$ & $95(6.4)$ & $<0.001$ \\
\hline
\end{tabular}

bDMARDs=biological disease-modifying antirheumatic drugs; CSs=corticosteroids; csDMARDs=conventional synthetic disease-modifying antirheumatic drugs; IL6i=interleukin- 6 inhibitor; NSAID=non-steroidal anti-inflammatory drug; T-cell=selective T-cell co-stimulation modulator; TNFi=tumor necrosis factor $\alpha$ inhibitor; P-values were calculated using Chi-square test

Conclusion: Patients with early RA treated with CSs in the first year after starting DMARDs tended to use more resources and have higher medical costs than patients not treated with CSs.

REFERENCES:

[1] Smolen JS et al., Ann Rheum Dis. 2020;79(6):685-699.

[2] JMDC claims database, Tokyo, Japan.

Disclosure of Interests: Eiichi Tanaka Speakers bureau: AbbVie GK, Asahi Kasei Pharma Corporation, Astellas Pharma Inc, Ayumi Pharmaceutical Corporation, Chugai Pharmaceutical Co., Ltd., Eisai Co., Ltd., Eli Lilly Japan K.K., GlaxoSmithKline K.K., Kyowa Pharma Chemical Co., Ltd., Janssen Pharmaceutical K.K., Mochida Pharmaceutical Co., Ltd., Pfizer, Takeda Pharmaceutical Co., Ltd, and Teijin Pharma Ltd., Eisuke Inoue Speakers bureau: Pfizer Japan, Bristol-Myers Squibb K.K., Ryoko Sakai Speakers bureau: Bristol Myers Squibb Co., Ltd., Grant/research support from: Tokyo Women's Medical University (TWMU), particularly the Division of Multidisciplinary Management of Rheumatic Diseases, Department of Rheumatology, has received unrestricted research grants from Ayumi Pharmaceutical Co.; Chugai Pharmaceutical Co., Ltd.; Eisai Co., Ltd., Nippon Kayaku Co., Ltd.; Taisho Toyama Pharmaceutical Co., Ltd.; Takeda Pharmaceutical Co., Ltd.; Mitsubishi Tanabe Pharma Co.; and Teijin Pharma Ltd., with which TWMU paid the salaries of RS., Iwasaki Katsuhiko: None declared, Ayako Shoji: None declared, masayoshi harigai Speakers bureau: AbbVie GK, Ayumi Pharmaceutical Corporation, Bristol-Myers Squibb K.K., Chugai Pharmaceutical
Co., Ltd., Eisai Co., Ltd., Eli Lilly Japan K.K., Pfizer Japan Inc., and Takeda Pharmaceutical Co., Ltd., Consultant of: AbbVie GK, Bristol-Myers Squibb K.K., Chugai Pharmaceutical Co., Ltd., Eli Lilly Japan K.K., and Gilead Sciences Inc., Grant/research support from: AbbVie GK, and Asahi Kasei Corp., Astellas Pharma Inc., Ayumi Pharmaceutical Corporation, Bristol-Myers Squibb K.K. Chugai Pharmaceutical Co., Ltd. Daiichi-Sankyo, Inc., Eisai Co., Ltd., Mitsubishi Tanabe Pharma Corporation., Nippon Kayaku Co., Ltd., Taisho Pharmaceutical Co., Ltd., and Takeda Pharmaceutical Co., Ltd.

DOI: 10.1136/annrheumdis-2021-eular.2805

\section{POS0555 THE NATURAL COURSE OF RHEUMATOID ARTHRITIS- ASSOCIATED INTERSTITIAL LUNG DISEASE FOCUSING ON LUNG PHYSIOLOGY: A PROSPECTIVE OBSERVATIONAL COHORT STUDY (PART 1)}

S. H. Chang ${ }^{1}$, J. S. Lee ${ }^{2}$, J. S. Lee ${ }^{3}$, C. H. Park' ${ }^{4}$, M. U. Kim ${ }^{5}$, Y. J. Ha ${ }^{6}$, E. H. Kang ${ }^{6}$, Y. A. Lee ${ }^{7}$, Y. Park ${ }^{8}$, J. Y. Choe ${ }^{9}$, E. Y. Lee ${ }^{10}$ on behalf of KOrean Rheumatoid Arthritis Interstitial Lung disease (KORAIL) cohort study group. ${ }^{1}$ Soonchunhyang University College of Medicine, Division of Rheumatology, Department of Internal Medicine, Cheonan, Korea, Rep. of (South Korea); ${ }^{2}$ Clinical Research Center, Asan Institute for Life Sciences, Asan Medical Center, Department of Medical Stastics, Seoul, Korea, Rep. of (South Korea); ${ }^{3}$ GENOME INSIGHT Inc.,., Seoul, Korea, Rep. of (South Korea); ${ }^{4}$ Soonchunhyang University College of Medicine, Department of Radiology, Cheonan, Korea, Rep. of (South Korea); ${ }^{5}$ SMG-SNU Boramae Medical Center, Department of Radiology, Seoul, Korea, Rep. of (South Korea); ${ }^{6}$ Seoul National University Bundang Hospital, Division of Rheumatology, Department of Internal Medicine, Seongnam, Korea, Rep. of (South Korea); ${ }^{7}$ Kyung Hee University College of Medicine, Division of Rheumatology, Department of Internal Medicine, Seoul, Korea, Rep. of (South Korea); ${ }^{8}$ Yonsei University College of Medicine, Division of Rheumatology, Department of Internal Medicine, Seoul, Korea, Rep. of (South Korea); ${ }^{9}$ Catholic University of Daegu College of Medicine, Division of Rheumatology, Department of Internal Medicine, Daegu, Korea, Rep. of (South Korea); ${ }^{10}$ Seoul National University College of Medicine, Division of Rheumatology, Department of Internal Medicine, Seoul, Korea, Rep. of (South Korea)

Background: Interstitial lung disease (ILD) is a severe extra-articular manifestation of rheumatoid arthritis (RA). However, there are few prospective studies for the natural course of lung physiology in most patients with RA-ILD.

Objectives: To assess the natural course of lung physiology of RA-ILD and the relation between arthritis activity and pulmonary physiology in patients with RA-ILD.

Methods: The KOrean Rheumatoid Arthritis ILd (KORAIL) cohort is the prospective observational cohort and aims to investigate the natural course of RAILD. Based on either 1987 or 2020 ACR criteria, patients diagnosed with RA and ILD based on CT scan were recruited from six tertiary medical hospitals in Korea since January 2015. RA disease activity was assessed using swollen and tender joint count by treating physician, inflammatory markers including CRP and ESR, and patient's global assessment annually. Pulmonary function tests (PFT), including FVC, FEV1, DLCO, and chest CT scan, were conducted annually.

Results: We analyzed 163 patients at baseline (V1), 141 at 1-year (V2), 122 at 2-year (V3), and 88 at 3-year follow-up (V4). The mean ( \pm SD) duration since RA diagnosis and since ILD diagnosis was $7.6 \pm 8.0$ and $2.7 \pm 3.1$ years, respectively. The female to male ratio was about $2: 1$, and $58.9 \%$ of patients $(n=96)$ were 65 years old or older. Only two patients were negative for RF and anti-CCP $98.7 \%$ of patients $(n=161 / 163)$ were positive for RF $(n=143,87.7 \%)$ or anti-CCP antibody $(n=154,94.5 \%)$. At enrollment, one-hundred-nine patients $(66.9 \%)$ had $\mathrm{FVC} \geq 80 \%$ of predicted. Twenty-five patients ( $15.3 \%$ ) showed FEV1/FVC $\geq 0.7$, of which seventeen patients, only ten percent of a total cohort (10.4\%), had FVC $\geq 80 \%$ of predicted, which corresponds to the obstructive pattern. Proportion of patients showed $\mathrm{a} \geq 10$-point decline from the enrollment in FVC of the predicted value were around $10 \%$ at every year (Table 1). The proportion of patients with a relative decline of $\geq 10 \%$ from the enrollment in FVC predicted was increased every year because of cumulation. The proportion of patients with a relative decline of $\geq 10 \%$ from the previous visit in FVC predicted was also around $10-15 \%$ every year. Proportions of patients with $55 \%$ or more DLco \% pred. has been decreased annually; $78.5 \%(n=128 / 158)$ at $V 1,72.9 \%(n=105 / 139)$ at $\mathrm{V} 2,68.7 \%$ $(n=90 / 117)$ at $V 3$, and $56.6 \%(n=56 / 85)$ at V4. Patients with a relative decline of $\geq 10 \%$ from the enrollment in DLco predicted was $38(27.5 \%)$ at V2 and 37 $(31.9 \%)$ at $\mathrm{V} 3$. Patients with a relative decline of $\geq 10 \%$ from the previous visit in DLco predicted was $29(25.2 \%)$ at V 3 . Of note, thirty patients $(21.7 \%)$ showed a relative $10 \%$ or more increase from enrollment in DLco predicted at V2, and so did 30 patients $(25.9 \%)$ at $\mathrm{V} 3$. 
Table 1. The analysis of forced vital capacity (FVC) in KORIL cohort

\begin{tabular}{|c|c|c|c|c|}
\hline & $\begin{array}{l}\text { V1 (enroll- } \\
\text { ment) }\end{array}$ & V2 (1-year) & V3 (2-year) & V4 (3-year) \\
\hline $\mathrm{FVC}(\mathrm{mL}), \operatorname{mean} \pm \mathrm{SD}$ & $\begin{array}{c}2549.5 \pm \\
743.3\end{array}$ & $\begin{array}{c}2479.5 \pm \\
764.4\end{array}$ & $\begin{array}{c}2435.8 \pm \\
742.8\end{array}$ & $\begin{array}{c}2405.7 \pm \\
731.2\end{array}$ \\
\hline FVC $\%$ of predicted, mean \pm SD & $\begin{array}{c}84.70 \pm \\
16.74\end{array}$ & $84.7 \pm 18.1$ & $84.3 \pm 17.6$ & $83.1 \pm 19.1$ \\
\hline Rate of $\mathrm{FVC}$ decline, $\mathrm{mL} \cdot \mathrm{year}^{-1}(95 \% \mathrm{Cl})$ & - & $-14(-49,21)$ & $-62(-104,-21)$ & - \\
\hline $\begin{array}{l}\text { A 10-point decline from V1 in predicted } \\
\text { FVC value, } n(\%)\end{array}$ & - & $10(7.1)$ & $16(13.3)$ & $8(9.4)$ \\
\hline $\begin{array}{l}\text { Relative decline of } 10 \% \text { from } \mathrm{V} 1 \text { in } \\
\text { predicted FVC value, } \mathrm{n}(\%)\end{array}$ & - & $17(12.1)$ & $23(18.9)$ & $17(19.3)$ \\
\hline $\begin{array}{l}\text { Relative decline of } 10 \% \text { from the previous } \\
\text { visit in predicted FVC value, } n(\%)\end{array}$ & - & $17(12.1)$ & $17(14.2)$ & $13(15.3)$ \\
\hline
\end{tabular}

Conclusion: Annually, about $10-15 \%$ of patients experience a relative $\geq 10 \%$ decline from the previous visit in FVC predicted value in RA-ILD.

Acknowledgements: This work was supported by the Korea Health Technology R\&D Project through the Korea Health Industry Development Institute, funded by the Ministry of Health and Welfare, Republic of Korea (grant no.HI14C1277). Disclosure of Interests: None declared

DOI: 10.1136/annrheumdis-2021-eular.2810

\section{POS0556 USEFULNESS OF FDG-PET/CT FOR PREDICTING SPONTANEOUS REGRESSION IN MTX ASSOCIATED LYMPHOPROLIFERATIVE DISORDER WITH RHEUMATOID ARTHRITIS}

T. Kameda ${ }^{1}$, S. Nakashima ${ }^{1}$, H. Shimada ${ }^{1}$, R. Wakiya ${ }^{1}$, M. Mahmoud Fahmy Mansour $^{1}$, M. Kato ${ }^{1}$, K. Sugihara ${ }^{1}$, Y. Ushio ${ }^{1}$, H. Dobashi ${ }^{1} .{ }^{1}$ Kagawa University, Department of Internal Medicine, Division of Haematology, Rheumatology and Respiratory Medicine, Faculty of Medicine, Kita-gun, Japan

Background: Recently, there are many reports from Japan about methotrexate associated lymphoproliferative disorder (MTX-LPD). We are investigating the predictive factor of spontaneous regression (SR) in MTX-LPD. On the other hand, FDG-PET/CT is used for diagnosis of LPD including malignant lymphoma. In addition, it was reported that imaging biomarkers such as metabolic tumor volume (MTV) and total lesion glycolysis (TLG) could predict the prognosis of malignant tumor $(1,2)$. However, there is no report that these imaging biomarkers could predict the SR of MTX-LPD.

Objectives: We investigate the usefulness of FDG-PET/CT for predictive factor of SR in MTX-LPD.

Methods: We enrolled 24 RA patients who diagnosed MTX-LPD and performed FDG-PET/CT from 2005 to 2019. We divided these cases into spontaneous regression cases (SR group; 15 cases) and cases that treated with chemotherapy after MTX discontinuation (CTx group; 9 cases), and compared the difference as follow subjects between two groups; clinical data including histopathological findings, SUVmax to evaluate malignant tumor activity by FDG-PET/CT, MTV and TLG which refer to metabolically active volume of the tumor segmented FDG-PET/CT. In addition, we analyzed cut off levels, sensitivity and specificity using statistical software JMP.

Results: Diffuse large B cell lymphoma (DLBCL) and Hodgkin lymphoma $(H L)$ were 5 and 1 cases in SR group, and 1 and 5 cases in CTx group. In addition, MTV and TLG by FDG-PET/CT was significantly lower in SR group, although SUVmax is no difference between two groups (figure 1). Cut off levels of MTV and TLG were $103.12 \mathrm{ml}$ (sensitivity; $88.9 \%$, specificity; $86.7 \%$ ) and $361.75 \mathrm{ml}$ (sensitivity; $88.9 \%$, specificity; $86.7 \%$ ), respectively.

Conclusion: We suggested that MTV and TLG were useful for predict of SR in MTX-LPD.

REFERENCES:

[1] Chen HH, Chiu NT, Su WC. et al. Prognostic value of whole-body total lesion glycolysis at pretreatment FDG PET/CT in non-small cell lung cancer. Radiology. 2012 Aug;264(2):559-66

[2] Chu KP, Murphy JD, La TH. et al. Prognostic value of metabolic tumor volume and velocity in predicting head-and-neck cancer outcomes. Int J Radiat Oncol Biol Phys. 2012 Aug 1;83(5):1521-7.
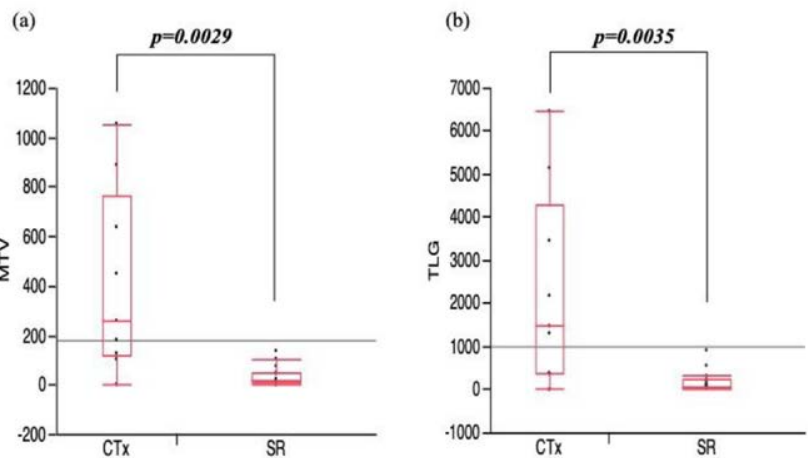

Figure 1. Comparison of the level of MTV(a) and TLG (b).

Disclosure of Interests: None declared

DOI: 10.1136/annrheumdis-2021-eular.2812

\section{POS0557 1 THE RELATION BETWEEN INVOLVEMENT OF INDIVIDUAL JOINTS OF THE UPPER EXTREMITY AND GRIP FORCE IN EARLY RHEUMATOID ARTHRITIS}

M. Rydholm ${ }^{1,2}$, A. Sharma ${ }^{2}$, I. Wikström ${ }^{1,2}$, L. T. H. Jacobsson ${ }^{2,3}, \underline{\text { C. Turesson }}{ }^{1,2}$ ${ }^{1}$ Skåne University Hospital, Department of Rheumatology, Malmö, Sweden;

${ }^{2}$ Lund University, Rheumatology, Department of Clinical Sciences, Malmö, Malmö, Sweden; ${ }^{3}$ Gothenburg University, Department of Rheumatology and Inflammation Research, Sahlgrenska Academy, Gothenburg, Sweden

Background: Rheumatoid arthritis (RA) has a major impact on hand function in many patients.

Objectives: The objective of this study was to investigate the relation between swelling and tenderness of individual joints in the upper extremities and grip force in patients with early RA.

Methods: An inception cohort of patients with early RA (symptom duration $\leq 12$ months), recruited in 1995-2005, was investigated and followed in a structured program. All patients were examined by the same rheumatologist according to a structured protocol, and swelling and tenderness of individual joints was recorded. Grip force (Newton, N) was measured using the electronic instrument Grippit (AB Detektor, Gothenburg, Sweden). Average grip force values of each hand were evaluated and compared to the expected, based on age- and sex-specific reference values from the literature (1). Grip force was expressed as \% of expected values. Linear regression analyses were used to assess the relation between upper extremity joint involvement in individual joints and grip force, using the presence/absence of synovitis/ tenderness in each joint as covariates. For the multivariate analysis, covariates with $p<0.10$ in the univariate analyses were selected. In cases with major collinearity (Spearman's $r>0.3$ ), the covariate with the stronger association in the univariate analysis was included.

Results: A total of 215 patients with early RA (71\% women; mean age 60 years) were investigated. The median symptom duration at inclusion was 7 months; interquartile range 5-10. The median 28 joint counts for swollen joints was 7 (interquartile range, IQR 5-11) and for tender joints 4 (IQR 1-9). In univariate analyses, swelling of the wrist, each metacarpophalangeal (MCP) joint and proximal interphalangeal (PIP) joints III and V, as well as tenderness of the elbow, wrist and each MCP and PIP joint were associated with significantly reduced grip force in the right hand. In the left extremity, corresponding associations were observed for swelling of the wrist, each MCP joint and PIP joints I and V, and for tenderness of the wrist and each MCP and PIP joint. In multivariate analysis, wrist synovitis was associated with reduced grip force ( $\beta-11.0$ and $-14.3 \%$ of expected, respectively). There were also independent significant associations for tenderness of MCP IV and the elbow with reduced grip force in the right hand, and for swelling of MCP I and MCP V with reduced grip force in the left hand (Table 1). 